\title{
Miranda
}

Revue pluridisciplinaire du monde anglophone /

Multidisciplinary peer-reviewed journal on the English-

speaking world

5 | 2011

South and Race / Staging Mobility in the United States

\section{Mobilité et immobilité dans les romans américains de Georges Simenon}

\section{Christine Calvet}

\section{OpenEdition}

Journals

Édition électronique

URL : http://journals.openedition.org/miranda/2462

DOI : 10.4000/miranda.2462

ISSN : 2108-6559

Éditeur

Université Toulouse - Jean Jaurès

\section{Référence électronique}

Christine Calvet, « Mobilité et immobilité dans les romans américains de Georges Simenon », Miranda [En ligne], 5| 2011, mis en ligne le 29 novembre 2011, consulté le 16 février 2021. URL : http:// journals.openedition.org/miranda/2462 ; DOI : https://doi.org/10.4000/miranda.2462

Ce document a été généré automatiquement le 16 février 2021.

\section{(c) (i) (5)}

Miranda is licensed under a Creative Commons Attribution-NonCommercial-NoDerivatives 4.0 International License. 


\title{
Mobilité et immobilité dans les romans américains de Georges Simenon
}

\author{
Christine Calvet
}

\section{Introduction}

1 Dans l'imaginaire d'un lecteur ou d'un spectateur, une intrigue de Georges Simenon renvoie plus volontiers aux bords du quai des Orfèvres, longés par la pipe et le chapeau mythiques du commissaire Maigret qu'à la vie sur le continent américain. Pourtant, dans l'abondante production écrite de l'écrivain belge et, bien que son œuvre forme un tout indissociable, on peut aisément repérer une période dite américaine. Elle correspond précisément à des textes rédigés pour la plupart entre 1945 et 1955. Mais c'est dès ses jeunes années que Simenon fait l'expérience de la mobilité puisqu'il finance, en 1935, grâce à son activité de journaliste, un premier tour du monde, en vendant ses reportages aux magazines et aux journaux.

2 Après la difficile période de la guerre qu'il a vécue en Europe, l'écrivain belge s'embarque pour l'Amérique où, toujours aussi productif, il écrit plus de quarante romans, dont une quinzaine retiennent plus particulièrement notre attention, puisque leur contenu fictionnel, tout comme celui de ses reportages publiés à la même époque, renvoie directement à sa perception du nouveau monde.

Du Canada à New York, du Connecticut au Nevada, bref, au fil de ses déplacements, Georges Simenon n'en finit pas de tracer la route de sa nouvelle vie, laissant tomber le rideau sur les scènes de guerre qui ont frappé la vieille Europe, cherchant à la fois l'oubli et la construction de ce que l'on pourrait appeler, en reprenant le titre d'un de ses romans, Une vie comme neuve ${ }^{1}$. Si le changement de cadre entraîne un changement de mode de vie radical, en revanche, sa méthode de création demeure inchangée de part et d'autre de l'Atlantique. Comme à l'accoutumée, ses capacités mémorielles lui permettent d'engranger de multiples sensations et de nombreux ressentis : New York, 
la Floride, la Louisiane, le Texas, l'Arizona, la Californie, constituent autant de terres où le romancier s'installe et prend racine pour un temps non déterminé et dont il repart subitement pour s'implanter dans un nouvel espace géographique. S'ensuit une sorte de sédimentation des contenus ainsi accumulés. C'est dans ce fonds-là que le romancier trouve les éléments nécessaires à la mise en place et au développement de ses intrigues. Aussi nous proposons-nous de fonder l'essentiel de notre réflexion sur le cadre fictionnel de trois romans particulièrement représentatifs de la période américaine de Georges Simenon. Les personnages du romancier, dans les diverses mises en scène de leurs vies quotidiennes, reflètent une forme d'identité américaine dont le miroir est en réalité constitué par les expériences vécues par Georges Simenon lui-même dans ses pérégrinations sur le continent nord-américain.

4 Les déplacements des individus occupent une place et une fonction prépondérantes dans l'économie des trois romans et entraînent le lecteur dans des espaces de vies multiples.

5 Le premier roman, Trois Chambres à Manhattan, paru en 1946, tient à l'évidence de l'autobiographie. L'intrigue se fonde en effet sur une passion amoureuse naissante entre deux êtres, directement inspirée du coup de foudre vécu par Georges Simenon et sa seconde femme, rencontrée à New York en $1945^{2}$. Le lecteur erre en noctambule dans les rues de Manhattan au rythme des pas du couple amoureux qui ne cesse de déambuler dans les artères de la ville. L'errance urbaine constitue en quelque sorte le moteur de l'action. Le second roman Feux rouges, paraît en 1953. Le lecteur quitte le réseau des rues rectilignes pour vivre, entre les états de New York et du Maine, l'interminable et éprouvant trajet d'un couple dont la voiture est prise dans un bouchon de quarante cinq millions d'automobilistes, expérience faite par Simenon luimême sur la route fédérale $\mathrm{N}^{\circ} 1$. La route n'est pas un simple ruban d'asphalte déroulé pour mettre en place un décor fictionnel, elle constitue un facteur de tension dramatique, puisqu'elle conduit le personnage principal de bar en bar, au point que l'alcoolisme qui ravage le couple entraînera sa rupture. La route n'est pas seulement un cadre, mais un élément déterminant de l'action. Le troisième roman, paru en $1947^{3}$, est moins directement autobiographique bien qu'à travers le regard de Maigret, que le romancier a emporté dans ses bagages, pointent les souvenirs de Georges Simenon débarquant dans la grande ville de New York. Sorte de théâtre dans le théâtre, les réactions du commissaire enquêteur-voyageur sont susceptibles de nous livrer un point de vue on ne peut plus franco-français sur l'univers policier américain.

6 Dans ces trois romans nous allons d'abord nous intéresser aux changements de décors et à leurs enjeux : le changement de continent entraîne-t-il un changement de décor radical? Les nouveaux cadres de vie mis en place sur la scène fictionnelle sont-ils en rupture avec les précédents? Quels en sont les éléments les plus représentatifs et peuton réellement parler de renouvellement? Ensuite nous analyserons les interactions qui se produisent entre les «espaces-temps » et les individus qui les habitent: la mobilité contribue-t-elle à « libérer » les personnages et peut-on considérer que les parcours accomplis sous les yeux du lecteur s'inscrivent dans ce qu'il est coutume d'appeler « le rêve américain»? Nous pourrons alors constater qu'en fait, dans les romans américains de Georges Simenon, la fiction ne résiste pas à une sorte cristallisation des mouvements humains et des déplacements identitaires: les individus se construisent dans les péripéties des crises qui les "agitent", et n'ont d'autre recours que de se mouvoir dans les marges étroites de leurs destinées. 


\section{Changement de continent, changement de décor}

En débarquant sur le continent américain, Simenon pénètre dans l'immensité d'un nouvel espace : neuf millions de kilomètres carrés sont à découvrir. Fidèle à son appétit de vie et à sa volonté de tout voir et tout connaître, il le parcourt de région en région. S'appropriant les «paysages", l'œil du romancier enregistre des éléments qu'il réinvestit inévitablement dans les romans rédigés pendant cette période. Si l'Amérique oriente incontestablement de manière nouvelle le regard du romancier, l'intériorisation de la vision propre à la fiction contribue à façonner une autre Amérique. Somme toute, on peut avancer qu'à tout point de vue, mémoire et imaginaire "déplacent les lignes »: des titres aux résonances d'un genre et d'un style inédits font leur apparition, comme par exemple, Maigret, Lognon et les gangsters ${ }^{4}$. C'est alors que le poêle ronflant des auberges françaises est détrôné par le ronflement de la vie américaine moderne avec ses highway, ses lumières néon, ses clubs de jazz si différents des guinguettes de Montmartre. La bière et l'absinthe sont remplacées par le whisky et le coca-cola.

Paradoxalement et en dépit de l'inflexion nouvelle que l'on peut déceler dans les romans de cette période - même si l'on sait que Simenon ne cherche jamais à imposer des conclusions - le lecteur peut s'étonner du peu de commentaires concernant la vie outre-Atlantique, de l'absence de jugements de valeurs et, finalement, de l'utilisation "globalisée et globalisante » de l'Amérique dans la matière fictionnelle. Cela s'explique en partie par l'attitude personnelle du romancier qui ne situe pas le drame social au centre de la littérature. Par ailleurs, Simenon n'écrit pas systématiquement des romans dont l'action se déroule à l'endroit où il vit. Un temps de décantation, lié à son mode de création, lui est nécessaire. Il mémorise nombre de ressentis, qui seront la matière d'une écriture constamment motivée non par le simple désir, mais par un besoin impérieux, une sorte d'urgence. Ainsi, bien que séjournant en Amérique, est-il capable d'écrire une intrigue se déroulant en plein Paris ou en Afrique. Le romancier est habité par une étonnante faculté de mobilité créatrice et ce qui fixe son attention est parfois totalement inattendu. A cela s'ajoutent une capacité descriptive efficace, rapide, héritée des années de journalisme, et une perception très sensitive des lieux et des gens. Et de fait, les éléments qui donnent une couleur américaine à certains de ses romans sont discrets tout en constituant un ensemble de détails, dont l'importance est précisée dans le reportage de 1946 intitulé L'Amérique en auto :

Je ne sais pas si vous êtes comme moi? Chaque fois que je lis une étude sur un pays que je ne connais pas, je me dis :

- Tout cela est fort intéressant. Mais comment est le vestibule, la cuisine, la salle à manger? Qu'est-ce que les gens mangent à leur déjeuner? A quoi ressemble le tramway qui les conduit à leur travail ?...

C'est peut-être naïveté de ma part, mais tous ces petits détails de la vie courante me manquent, le plus souvent, pour m'imaginer leur vie. Combien gagnent-t-ils? Combien payent-t-ils un kilo de pommes de terre ou un verre de bière ?... (L'Amérique en auto, 626)

Derrière la naïveté apparente, ces petits détails, observés dans la réalité quotidienne, fixent la vie dans le discours narratif. Le voyageur a toutefois une conscience aiguë de la richesse des multiples décors qui s'offrent à lui : 
Chacun qui débarque, après être passé devant la statue de la Liberté, peut, en quelque sorte, choisir l'Amérique qu'il désire. Il est à peu près sûr de la trouver. Que ce soient les faubourgs hantés de gangsters, les petites villes puritaines, les palais de milliardaires, les cafétérias où l'on se nourrit en rang devant un comptoir nickelé, que ce soient les plantations du Sud aux nègres nonchalants, les réserves d'Indiens et les lacs immenses, la fièvre de Broadway la nuit, ou la fièvre diurne de Wall Street, tout y est, de même que dans les magasins on trouve sans exception tout ce qui est annoncé sur le catalogue. (L'Amérique en auto, 626)

Simenon ne cherche pas à dresser un catalogue exhaustif des lieux traversés, ni à mettre en scène la réalité du quotidien américain, au contraire, il saisit quelques éléments dans leur nouveauté et, de manière contrastive, par touches impressionnistes, il en fait les témoins discrets de la spécificité culturelle avec laquelle il est en contact.

11 Le lexique, par exemple, s'enrichit, marquant de manière spécifique la description des décors. Apparaissent alors, pour n'en citer que quelques-uns, les termes de drive-in, cafeteria, juke-box, living corn, log cabin ou motel. Pareillement, la galerie des personnages se renouvelle, lorsque entrent en scène le détective privé, le gangster, le shérif ou la call girl..., qui « imprègnent » la fiction romanesque : dans Feux rouges, par exemple, le regard du narrateur se fixe sur l'emblématique et moderne appareil à musique des années trente : « Ici, il n'y avait pas de télévision, mais un juke-box lumineux, jaune et rouge, dont les rouages luisants maniaient les disques avec une fascinante lenteur.» (Feux rouges, 767).

12 Même si la trace d'une réelle admiration pour les objets de la vie moderne est perceptible dans le discours, leur caractère pittoresque est comme dilué dans les romans. Les objets ou les personnages nouveaux et typiquement américains subissent le même traitement que ceux de n'importe quel autre continent. Après la phase habituelle d'appropriation du milieu environnant et de ses spécificités, le romancier utilise la nouvelle pâte fictionnelle dont il dispose sans mettre l'accent sur les aspects emblématiques et les valeurs symboliques qu'elle contient, ainsi qu'en témoigne, de manière autobiographique, le personnage du commissaire Maigret devant le panorama de la ville de New York: «C'était sa première traversée, à cinquante-six ans, et il était tout étonné de se trouver sans curiosité, de rester insensible au pittoresque. » (Maigret à New York, 577).

13 A l'image de son personnage, Simenon se désintéresse du pittoresque. Bien au contraire, les lieux, quels qu'ils soient, se reflètent les uns les autres, ne serait-ce que par leurs traits communs, comme par exemple leur monotonie, caractéristique des mises en scène spatiales. Ainsi en est-il du «lieu-cliché » occupé par le couple amoureux de Trois Chambres à Manhattan :

L'appartement était tout petit, tout bête, un appartement comme il devait y en avoir des milliers à New York, avec le même cosy-corner dans le salon, les mêmes tables basses, les mêmes guéridons et les mêmes cendriers près des fauteuils, à portée de main, le même tourne-disque et la même bibliothèque minuscule dans un angle, près de la fenêtre. (Trois Chambres à Manhattan, 269-270)

14 En dépit de la modernité lexicale, le décor se dilue dans la répétition lancinante du " même ", n'affichant en définitive que l'image d'un conformisme sans grand intérêt. A bien considérer le standard émergeant de l'époque, le nouveau confort de la vie moderne qui place tout «à portée de main ", n'allège pas nécessairement le quotidien des personnages. Simenon suggère les limites des facilités apportées par le progrès et souligne la similitude des lieux, gommant leurs différences et les rendant étouffants et 
statiques, dans son discours même, sans rien commenter toutefois, laissant son « libre arbitre " au lecteur. Il n'hésite pas à mettre sur le même plan des lieux distants pour mettre en relief leur relative similitude. Dans Maigret à New York, figure une comparaison inattendue entre New York et une petite ville provinciale de la Loire ${ }^{5}$ :

Il pleuvait. On roulait dans un quartier sale où les maisons étaient laides à en donner la nausée. Etait-ce cela New York ?

Dix jours... Non, neuf jours avant, exactement, Maigret était encore installé à sa place habituelle, au Café du Cheval-Blanc, à Meung. Il pleuvait aussi, d'ailleurs. Il pleut aussi bien sur les bords de la Loire qu'en Amérique. (Maigret à New York, 580)

L'immensité de la ville de New York, son image grandiose et incomparable, sont réduites, dans l'esprit du personnage, à l'espace familier de la petite ville française et à la considération météorologique la plus plate.

Ainsi, l'univers américain simenonien échoue-t-il en partie dans sa fonction testimoniale : il ne livre pas d'indices originaux ou spectaculairement révélateurs quant aux lieux et aux comportements de vie du continent nord-américain, dont il serait cependant imprudent de sous-estimer l'importance, puisqu'ils peuvent être les véritables moteurs de l'action, occupant alors dans le récit une fonction narrative essentielle. Ainsi, dans Trois chambres à Manhattan, le romancier exploite-t-il les potentialités du réseau des rues de New York, pour transformer une rencontre en liaison passagère, puis en amour passionné. L'intrigue se noue dans l'espace de la rue :

Et ils y allèrent, de si bonne heure que le spectacle de la rue leur semblait plein de saveur inconnue. Sans doute avaient-ils déjà parcouru les rues l'un et l'autre de grand matin, mais ils ne l'avaient pas encore fait ensemble. Eux qui avaient tant traîné, la nuit, le long des trottoirs et dans les bars, ils avaient l'impression de se laver l'âme dans la frâicheur matinale, dans le débraillé allègre d'une ville qui fait sa toilette. (Trois Chambres à Manhattan, 269-270)

Notons que ce paragraphe pourrait correspondre au décor de n'importe quelle grande ville d'Amérique ou d'ailleurs. Il s'agit bien de New York, mais la ville n'est plus nommée en tant que telle; elle n'est même plus reconnaissable dans son évocation, et se trouve réduite à un simple pronom indéfini et au cliché universel d'une ville personnifiée qui se réveille.

18 Même lorsqu'il construit un " effet de réel » concernant « l'ailleurs » en utilisant des «localisateurs» spécifiques, Simenon n'exploite pas la veine exotique. Observons le personnage principal, François Combe, dans l'avenue new-yorkaise la plus célèbre :

Ce matin, il était sans amertume. Il avait marché jusqu'à Washington Square, où il avait pris l'autobus qui parcourt la Cinquième Avenue de bout en bout. Par goût, pour jouir du spectacle de la rue, il était monté sur l'impériale et il continuait, au début tout du moins, à se sentir allègre.

L'avenue était claire, les pierres des buildings d'un gris doré, donnant parfois l'illusion de la transparence, et le ciel, là-haut, d'un bleu pur, avec quelques petits nuages floconneux comme on en voit autour des saints sur les images pieuses. (Trois Chambres à Manhattan, 254)

19 Le personnage, arrivé dans la ville six mois auparavant seulement, semble s'être intégré à son nouvel espace de vie qu'il embrasse du regard le temps d'un trajet en autobus. Pourtant des modalisateurs atténuent la jouissance du «spectacle de la rue»: " semblait ", « au début tout du moins ", « illusion », autant de mentions qui annoncent que tout est trop beau pour être vrai. L'univers décrit est ramené à l'image d'un décor artificiel qui permet au romancier d'annoncer les difficultés à venir : ici, précisément, dans l'économie du récit, la peur du personnage de perdre son nouvel amour. La 
description du trajet s'achève sur une image stéréotypée qui fixe le sort de du personnage de Combe et le fige paradoxalement dans une forme d'immobilité, ou si l'on préfère dans l'immuabilité de son destin.

De la même manière, les motifs de la route et du trajet sont utilisés comme ressorts de dramatisation. Dans le reportage L'Amérique en Auto, Simenon rapporte les pénibles sensations vécues au cours d'un trajet effectué sur la route fédérale $\mathrm{N}^{\circ} 1$, finalement bloquée par un gigantesque bouchon :

Depuis Boston, je voyais la route devenir toujours plus large, mais je constatais aussi avec inquiétude qu'à chaque mille elle devenait plus infernale, littéralement noire de voitures trépidantes, de sorte qu'à la fin je finissais par conduire comme dans un rêve, dans un cauchemar plutôt, suivant le flot, me poussant à gauche ou à droite quand j'apercevais un trou dans la pâte mouvante. Les feux rouges, qui vous ordonnent impérieusement de stopper, les flèches indicatrices, est-ce que je les distinguais encore? Je fonçais, parmi des milliers d'autres voitures qui fonçaient tout autour de moi et j'avais conscience que, s'il m'arrivait par malheur de ralentir, toute la colonne m'entrerait dans le derrière. (L'Amérique en Auto, 649-650)

21 Ce phénomène de circulation massive n'a pas d'équivalent en son temps sur les routes européennes. Simenon saisit ici le fruit de son expérience personnelle et le réinvestit dans Feux rouges, non pour témoigner sociologiquement du phénomène en lui-même, mais pour utiliser le caractère hostile du milieu environnant qui étouffe son personnage et le pousse dans ses retranchements. Le décor fictionnel fige le mouvement: on se déplace, mais on n'avance pas. Le lecteur, qui suit le trajet du personnage, vit une succession d'événements au ralenti, la fluidité de la circulation étant constamment perturbée par l'affluence des automobilistes. Le décor est un agent direct de pénibilité tout au long du trajet : ce sont les conditions météo, ajoutées à la circulation difficile, qui mettent en place l'étau dans lequel le personnage va se trouver pris :

Ce qui tendait ses nerfs, c'était le bruit obsédant des roues des deux côtés, les phares, qui, de cent mètres en cent mètres, se précipitaient à sa rencontre, c'était aussi la sensation d'être prisonnier dans le flot, sans possibilité de s'échapper à gauche ou à droite, ou même de ralentir, car son rétroviseur lui montrait un triple chapelet de lumières qui le suivaient pare-chocs à pare-chocs. (Feux rouges, 754-755)

Alors que l'œil du reporter se fixe sur les faits et les événements réels, celui du romancier donne toute sa force poétique au souvenir et pousse l'utilisation du cadre de la route jusqu'à son paroxysme, passant du réel à l'onirique :

Les enseignes au néon avaient commencé à surgir sur la droite où, avec les pompes à essence, elles constituaient les seuls signes de vie. Sans elles, on aurait pu croire que la grand-route était suspendue dans l'infini et qu'au-delà n'existaient que la nuit et le silence. Les villes et les villages étaient tapis plus loin, invisibles, et ce n'était que rarement qu'un vague halo rougeâtre dans le ciel laissait deviner leur existence.

La seule réalité proche, c'étaient les restaurants, les bars qui jaillissaient du noir tous les cinq ou dix miles, avec, en lettres rouges, vertes ou bleues, le nom d'une bière ou d'un whisky. (Feux rouges, 754-755)

La route devient un ressort de la dramatisation, un espace imprécis et déstabilisant : sur une scène dangereuse un personnage oppressé, de verre d'alcool en verre d'alcool, va perdre les pédales. "Les phares se précipitent ", «les néons surgissent », ce sont donc les éléments du décor qui mènent le jeu, et qui, par le choix de la voix active, leur donnent le statut de sujet pilotant les événements. Le conducteur passe au second plan et subit la situation. Le décor devient une instance autonome active, un acteur de 
l'action qui «manipule » les personnages. Nous allons regarder de plus près comment s'opèrent ces manipulations, ou, plus exactement, comment se développent les interactions entre les « espaces-temps » et les personnages qui les « habitent ».

\section{Interactions entre " espaces-temps » et personnages} principaux. Dans Trois chambres à Manhattan, Combe parcourt inlassablement le réseau des rues; dans Feux rouges, le couple des parents, Nancy et Steve, effectue plusieurs centaines de kilomètres pour aller chercher ses enfants dans un camp de vacances au sud du pays; dans Maigret à New York, le casanier commissaire traverse pour la première fois l'Atlantique. Les déplacements des personnages, dans tous les cas, prennent dans la fiction une dimension expérimentale. Combe, tout en réalisant son parcours amoureux, cherche à construire son identité; les parents en difficulté sont mis à l'épreuve dans un embouteillage qui accentue les tensions au point de séparer le couple; le commissaire, dépaysé, mène son enquête en terre américaine, et ses déplacements le conduisent à découvrir peu à peu les fondements de la justice et les méthodes de la police outre-Atlantique. Simenon observe les réactions de ses personnages dans leur milieu environnant à la manière d'un biologiste examinant la forme que prend la vie dans une boîte de Pétri. En les plongeant dans un univers inconnu, il rend compte des interactions entre les décors, les "paysages » et leurs comportements, cherchant à mettre à nu leur intériorité. Plus qu'à l'aspect matériel de l'environnement, il s'intéresse à «l'humain ».

Comme c'est fréquemment le cas dans l'intrigue simenonienne, le personnage principal se sent mal à l'aise et éprouve le besoin irrésistible de bouger. Le commissaire Maigret lui-même, ne supportant pas le dépaysement, a du mal à s'orienter dans New York et il finit par quitter l'hôtel luxueux dans lequel il a été accueilli pour gagner un lieu plus modeste et plus populaire. Au cours d'une conversation téléphonique, il répond à son confrère américain, le capitaine O'Brien :

Mais oui, j'ai trouvé un hôtel. Le Berwick. Vous ne connaissez-pas? Je ne sais plus le numéro de la rue. Je n'ai jamais eu la mémoire des chiffres et vous êtes ennuyeux avec vos rues numérotées. Comme si vous ne pouviez pas dire rue Victor-Hugo, rue Pigalle ou rue du Président je ne sais qui... (Maigret à New York, 605)

Le personnage perd ses repères lorsqu'il quitte son univers d'origine: il tente de s'adapter à la structure géographique et à l'atmosphère de la ville de New York en gagnant un lieu qui lui correspond mieux. Dès qu'il parvient à reconstruire une ambiance un peu plus familière, le malaise s'estompe :

Il était de bonne humeur, et même d'humeur enjouée, sans raison bien précise, peut-être simplement parce qu'il se retrouvait dans une atmosphère qui lui était familière.

D'abord, il aimait ce coin bruyant et un peu vulgaire de Broadway, qui lui rappelait à la fois Montmartre et les grands boulevards de Paris. (Maigret à New York, 606)

[...]

Le Berwick, déjà, l'avait réconcilié avec l'Amérique, peut-être à cause de son odeur d'humanité, et maintenant il imaginait toutes les vies tapies dans les alvéoles de ces cubes de briques, toutes les scènes qui se déroulaient derrière les stores. (Maigret à New York, 610) 
obilité nécessaire à Maigret pour reconstruire un cadre de vie plus familier et plus modeste ne gêne personne dans un pays où l'on peut aller et venir, et s'installer à sa guise. En revanche, la mobilité excessive de la population d'un lieu de vie à un autre, quant à elle, entrave la méthode d'investigation du commissaire. En effet, le mode de vie des américains provoque un déracinement constant et ne permet guère de percer un anonymat omniprésent. Dans les quartiers populaires, les habitants ne se fixent pas durablement, déménagent fréquemment, comme le constate le commissaire lors des traditionnels interrogatoires qui jalonnent une enquête :

- Savez-vous si, dans la maison, il y a encore des locataires qui s'y trouvaient déjà il y a une trentaine d'années?

On fronçait les sourcils, car c'était bien là la question à laquelle on s'attendait le moins. A Paris, à Montmartre par exemple, ou bien dans le quartier qu'il habitait, entre République et Bastille, il n'existait peut-être pas un immeuble de quelque importance où il n'eût trouvé aussitôt une vieille femme, un vieil homme, un couple installé dans la maison depuis trente ou quarante ans.

Ici, on lui répondait :

- Il n'y a que six mois que nous sommes arrivés...

Ou un an, ou deux. Le maximum était quatre ans. (Maigret à New York, 624)

La vie des locataires entassés se fige sur elle-même et devient étanche au monde extérieur.

Pourtant, dans ses reportages, Simenon ne souligne pas son enthousiasme devant la capacité d'assimilation de la population étrangère dans et par le « Nouveau Monde » :

Vous êtes ici? Bon. C'est que vous avez le droit d'y être. Vous bénéficierez automatiquement du préjugé favorable. Ce n'est pas à vous de prouver que vous êtes en règle, ou que vous êtes innocent. C'est aux autorités de prouver que vous êtes coupable.

Vous êtes norvégien, russe, français, italien? Vous ne parlez que votre langue natale? A votre aise. Tirez vos plans. Personne ne vous empêche d'ouvrir boutique si bon vous semble ou de choisir n'importe quelle profession.

Parce qu'au fond, l'Amérique sait bien que, dans un peu de temps, quelle que soit votre race, vous serez un Américain cent pour cent parce qu'elle a confiance en votre force d'assimilation. (L'Amérique en Auto, 685)

Dans la fiction romanesque, les choses paraissent moins faciles pour les personnages qui, bien que parvenant à s'adapter, ne sont pas réellement assimilés. Cette contradiction apparente correspond à la logique de deux écritures. Tandis que le journaliste jette un coup d'œil sur la vie réelle, le romancier la scrute d'un regard lucide et distancié, dans la constante préoccupation d'en exploiter les éléments à des fins purement narratives: dans le roman, Simenon en reste au simple constat, n'utilisant le fait de société que pour freiner le rythme de l'enquête.

Le commissaire rencontre des difficultés, liées aux conditions de vie des Newyorkais, pour mener son enquête à son terme, mais, au bout du compte, avec l'expérience des hommes qui est la sienne, il s'accommodera. Il se montrera même capable, lui qui n'aime pas changer de lieu de vie, de goûter le soleil américain avec plaisir :

Pour la première fois, depuis qu'il avait débarqué à New York, il était accueilli à son réveil par un soleil vraiment printanier : il en pénétrait un petit bout dans sa chambre et dans sa salle de bains.

A cause de ce soleil, d'ailleurs, il avait accroché son miroir à l'espagnolette de la fenêtre, et c'était là qu'il se rasait, comme à Paris, boulevard Richard Lenoir, où, le matin, il avait toujours un rayon de soleil sur la joue quand il se faisait la barbe. N'est-ce pas une erreur de croire que les grandes villes sont différentes les unes des 
autres, même quand il s'agit de New York, que toute une littérature représente comme un sorte de monstrueuse machine à malaxer les hommes?

Il y était, à New York, lui, Maigret, et il y avait une espagnolette à la bonne hauteur pour se raser, un rayon de soleil oblique qui lui faisait cligner de l'œil et, en face, dans des bureaux ou des ateliers, deux jeunes filles en blouse blanche riaient de lui. (Maigret à New York, 646) de rêve, reçoit un traitement inattendu dans les romans de Simenon. Non seulement, le romancier en néglige les éléments qui permettraient de restituer le pittoresque, mais il fond les différents lieux qu'il utilise en une sorte de pâte fictionnelle indifférenciée, au point que leurs descriptions pourraient sans difficulté prendre place dans n'importe lequel de ses romans. Les villes, les quartiers, les logements, les objets du quotidien semblent se cristalliser pour ne constituer qu'un espace global où l'homme, quel qu'il soit, tente de «mener sa vie ». Ainsi, les lieux de vie rappellent-ils d'autres lieux de vie, les hommes qui vivent dans des microcosmes rappellent d'autres hommes vivant dans d'autres microcosmes. En plein New York, par exemple, Maigret pénètre dans le quartier de Greenwich Village, qui constitue une sorte de «réduction» de la grande ville :

C'était lui qui lui avait donné au taxi une adresse dans Greenwich Village et Maigret découvrait, au cœur de New York, à quelques minutes des buildings, une petite ville encastrée dans la ville, une cité quasi provinciale, avec ses maisons pas plus hautes qu'à Bordeaux ou à Dijon, ses boutiques, ses rues calmes où l'on pouvait flâner, ses habitants qui ne paraissaient pas se soucier de la cité monstrueuse qui les entourait. (Maigret à New York, 646)

Et lorsqu'il pénètre dans l'appartement d'un particulier :

Et on avait l'impression d'accomplir, rien qu'en franchissant l'étroit obstacle d'un paillasson troué, un immense voyage dans l'espace et le temps. On n'était plus à New York, à deux pas des gratte-ciel qui, à cette heure, jetaient tous leurs feux dans le ciel de Manhattan. (Maigret à New York, 636) 

valeur générale à son discours. Greenwich Village est un monde dans un monde, un repli du décor sur le décor d'une petite humanité dont l'appartenance géographique n'a plus guère d'importance. La concentration de l'espace et du temps révèle la relative uniformité de mondes qui entrent en résonance.

La mobilité des personnages est conditionnée par le milieu ambiant qui, paradoxalement la détermine et l'entrave systématiquement. Les personnages bougent, tentent de s'adapter et de prendre place dans un "espace-temps ", où en dépit du mouvement et $\mathrm{du}$ nombre, la solitude triomphe. Le narrateur utilise le regard du commissaire Maigret, en focalisation interne, pour constater :

Rares étaient les gens qui se connaissaient d'étage à étage. [...] Les paliers, qui étaient vastes, constituaient en quelque sorte des terrains neutres où l'on entassait tout ce qui ne servait pas dans les logements. [...] Il était difficile de concentrer plus de vies humaines dans aussi peu d'espace et pourtant on ne sentait aucune chaleur, on éprouvait plus que nulle par ailleurs un sentiment d'irrémédiable isolement. (Maigret à New York, 625)

La «solitude dans la multitude ${ }^{6}$ reste une constante, même si l'on vit dans un Nouveau Monde où tout semble possible, comme en témoigne, toujours en focalisation interne, Trois Chambres à Manhattan :

Et tout ce qu'il voyait autour de lui, ce pèlerinage dans un monde de grisaille, où des hommes noirs s'agitaient dans le rayon des lampes électriques, ces magasins, ces cinémas avec leurs guirlandes de lumières, ces boutiques à saucisses ou à pâtisseries écœurantes, ces boîtes à sous, à musique, à lancer des billes dans des petits trous, tout ce qu'une grande ville a pu inventer pour tromper la solitude des hommes, tout cela, il pouvait le regarder enfin, désormais, sans écœurement ni panique.

[...] Une seule angoisse encore, l'ultime, qu'il traînait avec lui de bloc de maisons en bloc de maisons, ces cubes de briques le long desquels courent des escaliers de fer, pour les cas d'incendie, et dont on se demande, non pas comment les gens ont le courage d'y vivre, ce qui est encore assez facile, mais le courage d'y mourir. (Trois Chambres à Manhattan, 316)

Simenon exploite le cadre américain et le foisonnement de vie qu'il semble proposer comme une variation sur ses thèmes de prédilection. Le changement de cadre nourrit son imaginaire, il n'est qu'un alibi pour réactiver des préoccupations de type universel, comme la solitude des hommes ou la pesanteur de la mort. Les escaliers de fer, qui illustrent la volonté de l'homme de sécuriser les lieux de vies et devraient constituer une vision rassurante, polluent au contraire le cadre de vie. Objets récurrents dans les décors américains de Simenon, ils sont aussi l'objet de l'attention de Maigret, évoquant davantage la descente vers la mort et l'enfer que la garantie de la vie :

Bientôt, le long des rues rectilignes, interminables, on ne vit plus guère circuler que des gens de couleur. C'était Harlem qu'on traversait, avec ses maisons toutes pareilles les unes aux autres, ses bloc de briques sombres qu'enlaidissaient par surcroît, zigzagant sur les façades, les escaliers de fer pour les cas d'incendie.

On franchissait un pont, beaucoup plus tard. On frôlait des entrepôts ou des usines - il était difficile de distinguer dans l'obscurité - et c'était, dans le Bronx, de nouvelles avenues désolées, avec parfois les lumières jaunes, rouges ou violettes d'un cinéma de quartier, les vitrines d'un grand magasin encombrées de mannequins de cire aux poses figées.

On roula plus d'une demi-heure et les rues devenaient toujours plus sombres, plus désertes, jusqu'à ce qu'enfin le chauffeur arrêtât sa machine et se retournât en laissant tomber d'un ton dédaigneux : - Findlay.

[...] Dans la perspective noire, on voyait se découper le rectangle de quelques 
boutiques comme il en existe dans les quartiers pauvres de Paris et de toutes les capitales. (Maigret à New York, 610) en petits morceaux de vie devenant de simples matériaux uniformes. Le monde n'est plus qu'une vaste scène où les visages deviennent des masques, portant le poids de la résignation.

Et des tramways passaient, pleins de visages livides et secrets. Et des enfants, de petits bonhommes tout noirs dans le gris, rentraient de l'école en s'efforçant, eux aussi, d'atteindre à la gaieté.

Et tout ce qu'on voyait dans les vitrines était triste. Et les mannequins de bois ou de cire avaient des poses hallucinantes, tendaient leurs mains trop roses en des gestes d'inadmissible acceptation. (Trois Chambres à Manhattan, 316)

41

Les citadins se déplacent dans « leur » tramway, accomplissant des trajets répétitifs qui les fixent dans leur quotidienneté. Automatisés dans une vie au jour le jour, sans surprise, résignés, ils sont peu différents des mannequins des vitrines et vivent sans réelle prise sur l'orientation et le sens de leur existence.

Pourtant, le Capitaine O'Brien rappelle à Maigret combien on se soucie du bonheur des hommes, en respectant le principe de la liberté individuelle, notamment celle de se déplacer, qu'il s'agisse de mobilité physique ou sociale. Il argumente auprès de Maigret :

Les formalités pour entrer aux Etats-Unis ont peut-être été longues mais du moins, une fois terminées, notre homme est absolument libre.

- Saisissez-vous?

- Tellement libre qu'à moins qu'il tue, qu'il vole ou qu'il viole, nous n'avons plus le droit de nous occuper de lui. (Maigret à New York, 598)

Ce souci du respect et de la protection de la liberté individuelle s'inscrit pourtant dans une inexorable fatalité. L'utilisation par le romancier de la circulation et de ses dangers, n'est pas seulement emblématique du Nouveau Monde, mais symbolique : elle traduit l'imminence de la mort à chaque tournant de la vie. Dans Feux rouges, la route devient un univers non maîtrisé, présenté comme dévorant des vies en quantité monstrueuse, et les statistiques annoncées sur les ondes radio ont le ton de la fatalité :

Un monsieur sans veston, sur l'écran de la télévision, aux lunettes à grosse monture d'écaille qui paraissaient donner chaud, annonçait d'un ton de morne conviction :

- Le National Safety Council prévoit pour ce soir de quarante à quarante-cinq millions d'automobilistes sur les routes et évalue à quatre-cent trente cinq le nombre de personnes qui, d'ici, lundi soir, perdront la vie dans des accidents de la circulation. Il continuait, lugubre, avant d'être remplacé par une réclame de bière :

- Evitez d'en être. Soyez prudents. (Feux rouges, 750)

Derrière l'humour, Simenon met en scène le destin. Sur tous les continents, dans tout le roman, lui seul, à l'image de ce qu'il constate partout dans la vie réelle, décide du sort et du mouvement des hommes. Les romans américains de Simenon ne sont, en ce sens, pas différents de l'ensemble de son œuvre. Il écrit à propos de la création romanesque :

Je veux qu'un livre ait la même résonance à New York ou à Boston qu'à Londres, à Liverpool et à Paris. Tant qu'un roman n'a pas obtenu ce degré de vie là, dans six ou sept pays au minimum, à mes yeux, il n'a pas fini de naître. (Klinkenberg, 133)

Simenon se sert de la nouveauté apportée par le continent américain pour poursuivre son inlassable quête de «l'homme nu ». Ses personnages, si désireux d'échapper à leur mal-être, si avides de se construire ailleurs une "nouvelle vie ", toujours prêts à fuir, ignorants le plus souvent que c'est eux-mêmes qu'ils fuient, sont en réalité figés dans ce 
qui ne peut être que leur destinée. Ils sont paradoxalement immobiles, et leur mobilité, si visible, comme l'agitation ou les reflets à la surface de l'eau, recèle en profondeur une incontestable forme d'immuabilité. En définitive, l'étude de la mobilité, le suivi des déplacements pour une compréhension de parcours divers permet au romancier de mettre à nu les crises internes de personnages confrontés malgré eux à l'inexorabilité de leur destin.

\section{Conclusion}

Du simple point de vue biographique, il est certain que Georges Simenon a subi l'attraction du Nouveau Monde, au point d'envisager un temps de demander la nationalité américaine. Mais le romancier n'a en fait pas d'autre identité que celle de créateur des mondes et des personnages de son œuvre. Sa vie durant, il a poursuivi le même projet : rendre compte de "l'homme nu ", quels que soient son milieu social, sa culture, sa langue, son implantation géographique ou ses lieux de séjour. L'ensemble de ses romans vise à construire l'identité universelle de l'homme tel qu'il est, partout et toujours.

A son retour d'Amérique, il continuera à écrire des romans sans pour autant se référer particulièrement à l'expérience vécue outre-Atlantique. Peu importe le décor dans lequel se déroule la fiction et l'influence de ce décor sur la vie des personnages mis en scène, seul compte "l'homme ", dans son immobilité profonde, pris aux pièges de ses incessants déplacements et condamné à vivre et mourir en scène, dans la tragédie de sa destinée.

Pour ce qui est de la poétique du roman simenonien, elle est beaucoup plus complexe qu'il est coutume de le dire, et les romans dits américains donnent de l'épaisseur à de nombreuses interrogations. Assurément le behaviourisme est présent dans l'œuvre de Simenon, sous la forme d'une recherche constante de compréhension des comportements humains, soigneusement observés, et l'ensemble des romans constituent une vaste mosaïque, voire une fresque, cohérente. Les procédés du roman réaliste et naturaliste sont utilisés, certes, notamment pour construire des « effets de réel », mais Simenon n'a personne pour « modèle privilégié », ni Zola, ni Maupassant, ni même Dostoïevski, malgré de très réelles influences. En revanche, son exploitation des ressentis et des souvenirs, sa prospection des analogies, son travail d'écriture qui confronte souvent mémoire et imaginaire, rappellent parfois étrangement la démarche proustienne. Sa conception de l'homme, loin de toute prise d'engagement " existentialiste", est bien proche des constats de Camus. Quant à sa poétique, elle emprunte autant à la poésie qu'à la prose narrative. Comment ne pas songer à Baudelaire, à cet «Amer savoir» "qu'on tire du voyage », ou encore à la chute des «Hiboux $»^{8}$ :

Leur attitude au sage enseigne

Qu'il faut en ce monde qu'il craigne

Le tumulte et le mouvement ;

L'homme ivre d'une ombre qui passe

Porte toujours le châtiment

D'avoir voulu changer de place. (Baudelaire, 67) 


\section{BIBLIOGRAPHIE}

Baudelaire Charles. Les Fleurs du Mal. [1861] : Paris : Gallimard, La Pléiade, tome I, 1971.

---, Le Spleen de Paris. [1869]. Paris : Gallimard, La Pléiade, tome I, 1971.

Carly, Michel. Sur les routes américaines avec Simenon. Paris : Carnet Omnibus, 2002.

Klinkenberg, Jean-Marie. Lire Simenon. Réalité, fiction, écriture. In reCollectif, Bruxelles : Ed. Labor, $1980,117-142$.

Simenon, Georges. Trois Chambres à Manhattan [1946]. Paris : Omnibus, tome I, 2002, 209-324.

---, Maigret à New York [1946]. Paris : Omnibus, Tout Simenon, tome I, 2002, 577-684.

---, Feux rouges [1953]. Paris : Omnibus, Tout Simenon, tome VI, 2002, 747-849.

---, L'Amérique en auto, Mes apprentissages. Reportages, 1931-1946. Paris : Omnibus, 2001, 625-693.

\section{NOTES}

1. Simenon, Georges, Une Vie comme neuve. [1951]. Paris: Omnibus, Tout Simenon, Tome 5, 2002.

2. Il s'agit de Denise Ouimet que Simenon épousera en 1950.

3. La rédaction et la publication, dans la plupart des cas, se succèdent sans long délai. Généralement, le roman achevé paraît quelques mois seulement après la fin de la rédaction.

4. Maigret, Lognon et les gangsters, Paris : Presses de la Cité, 1952.

5. Meung sur Loire est une ville de 6000 habitants environ, à notre époque.

6. Baudelaire, Charles, Le Spleen de Paris, Les Foules, XII, 291-292.

7. Baudelaire, Charles, Les Fleurs du Mal, Le Voyage, CXXVI, 133.

8. Baudelaire, Charles, Les Fleurs du Mal, Les Hiboux, LXVII.

\section{RÉSUMÉS}

Dans l'abondante production de Georges Simenon, on peut aisément repérer une période dite américaine, de 1945 à 1955, qui correspond aux dix années passées en Amérique durant lesquelles l'écrivain belge écrira une quarantaine de romans. Après la guerre, Simenon cherchera en effet l'oubli et la construction d'Une vie comme neuve : changement de cadre et changement radical de mode de vie, mais la méthode demeure inchangée. Au fil des déplacements, la mémoire enregistre de multiples sensations qui se sédimentent pour constituer le fonds dans lequel puise le romancier et c'est le cadre fictionnel de trois romans de cette période américaine qui sont au cœur de cette étude : Trois chambres à Manhattan, (1946), Feux rouges (1953) et Maigret à New York 
(1946). Dans ces trois ouvrages, au caractère autobiographique marqué, l'analyse porte sur les spécificités et les enjeux des changements de décor, puis sur les interactions entre les personnages et les «espaces-temps » qu'ils habitent. De manière paradoxale, il ressort que «mobilité » et « immobilités» sont les deux paramètres d'une "immuabilité fondamentale » celle du destin tragique de «l'homme nu».

Within Georges Simenon's abundant production, a so-called American period is readily identifiable. It corresponds to the years 1945 to 1955, which the Belgian writer spent in America and during which he wrote approximately forty novels. After the war, Simenon sought to forget, aspiring to A New Lease of Life: a change of surroundings and a radical change of lifestyle. His method, however, remained unchanged. From one journey to another, Simenon's memory recorded feelings and impressions, forming sediment on which the novelist subsequently drew. The fictional framework of three novels of this American period, Trois Chambres à Manhattan, (1946), Feux rouges (1953) and Maigret à New York, is the object of the present study. The analysis of these three markedly autobiographical works focuses on the specificities and of the changes of scenery and their mechanism, then on the interactions between the characters and the "spacetimes" in which they live. Paradoxically, "mobility» and «immobilities" are the two parameters of a « fundamental immutability », that of the tragic destiny of « the naked man ».

\section{INDEX}

Mots-clés : cristallisation, conformisme, décor, destinée, espace américain, fiction, identité, imaginaire, immobilité, itinéraire, mise en scène, mobilité, modernité, narration, parcours, paysage, pittoresque, réalité, récit autobiographique, rêve américain, roman policier, tragique, ville

Keywords : crystallization, conventionality, scenery, fate, American space, fiction, identity, imaginary, immobility, itinerary, staging, mobility, modernity, narration, route, landscape, picturesque, reality, autobiographic narrative, American dream, detective story, tragedy, city

\section{AUTEURS}

\section{CHRISTINE CALVET}

Ingénieur d'étude

Université Toulouse 2 - Le Mirail

christine.calvet@univ-tlse2.fr 